\title{
Catalytic asymmetric S-H insertion reaction of carbenoids
}

\author{
Xiaomei Zhang, Ming Ma and Jianbo Wang*
}

Key Laboratory of Bioorganic Chemistry and Molecular Engineering of Ministry of Education, Department of Chemical Biology, College of Chemistry, Peking University, Beijing 100871, P. R. China

E-mail: wangjb@pku.edu.cn

\section{This paper is dedicated to Professor Zhi-Tang Huang on the occasion of his $75^{\text {th }}$ birthday}

(received 17 Nov 02; accepted 28 Jan 03; published on the web 19 Feb 03)

\begin{abstract}
Catalytic asymmetric S-H insertion of carbenoids generated from aryldiazoacetates has been investigated with a number of chiral $\mathrm{Rh}(\mathrm{II})$ and $\mathrm{Cu}(\mathrm{I})$ catalysts. $12 \%$ - $23 \%$ ee enantioselectivity were achieved with chiral Rh(II) catalysts.
\end{abstract}

Keywords: S-H Insertion, carbenoids, catalytic asymmetric synthesis

\section{Introduction}

Optically active $\alpha$-mercapto carboxylic acid derivatives are ubiquitous structural subunits in numerous biologically active natural and unnatural compounds. Compounds containing the mercapto or mercaptoacyl moiety often exhibit strong inhibitory effects on metal-containing enzymes (metallozymes). For example, $\alpha$-mercaptoacyl dipeptides QS-26332 and BMS-182657, as shown in Scheme 1, have been demonstrated to be potent inhibitors of angiotensin converting enzyme (ACE) and neutral endopeptidase. ${ }^{1}$ Consequently, the synthesis of enantioenriched $\alpha$-mercapto carboxylic acids attracted considerable attention. ${ }^{2}$ Although some methods have been developed, the stereoselective synthesis of these compounds still remains to be a formidable challenge. To the best of knowledge, so far there has be no highly selective catalytic asymmetric method for preparing enantioenriched $\alpha$-mercapto carbonyl compounds.

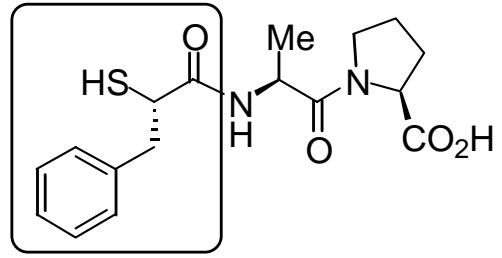

SQ-26332

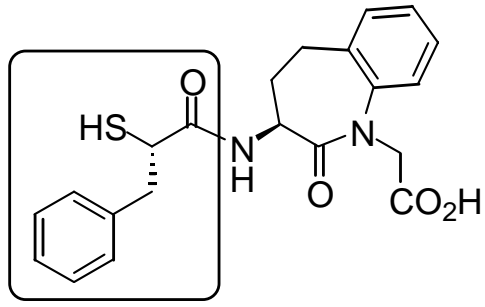

BMS-182657

\section{Scheme 1}


The direct S-H insertion of $\alpha$-diazocarbonyl compounds with thiol provides an efficient route to $\alpha$-mercapto carbonyl compounds. ${ }^{3}$ It would be highly desirable if the stereochemistry of the S-H insertion can be controlled by the chiral ligands of the metal catalysts. As far as our knowledge is concerned, there is only one report about the investigation in this area. Brunner and Doyle reported the S-H insertion reaction of 3-diazobutan-2-one with thiophenol in the presence of chiral $\mathrm{Cu}(\mathrm{I})$ and $\mathrm{Rh}(\mathrm{II})$ catalysts. Up to $13.8 \%$ ee was achieved. ${ }^{4}$ Since the carbenoids derived from aryldiazoacetate shown exceptionally high enantioselectivity in C-H insertion ${ }^{5}$ and moderately high enantioselectivity in sulfur ylide [2,3] sigmatropic rearrangement, ${ }^{6}$ we reasoned that it would be worthwhile to investigate the corresponding catalytic asymmetric S-H insertion with aryldiazoacetates. In this paper, we present our investigation along this line (Scheme 2).

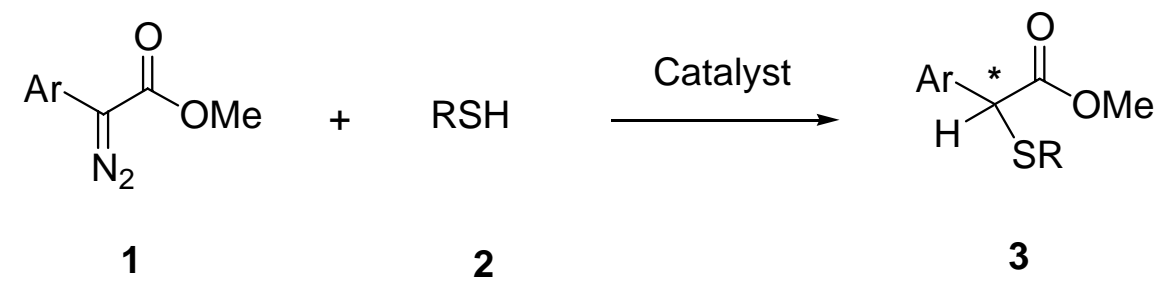

\section{Scheme 2}

\section{Results and Discussion}

Firstly, the phenyldiazoacetates was employed as the diazo substrate to optimize the reaction conditions. A wide range of chiral catalysts was selected for study (Scheme 3). These chiral catalysts have been demonstrated to be highly effective in asymmetric reaction of carbenoids, such as C-H insertion and cyclopropanation. ${ }^{7}$ From the results summarized in Table 1, it can be seen that the decomposition of diazo compounds in the presence of chiral $\mathrm{Rh}(\mathrm{II})$ and $\mathrm{Cu}(\mathrm{I})$ catalysts gave the expected S-H insertion product in moderate yields, but the enantioselectivities were low. $\mathrm{Cu}(\mathrm{I})$ catalysts are generally less effective than $\mathrm{Rh}(\mathrm{II})$ catalysts (Table, entries 1-9). The reactions with $\mathrm{Cu}(\mathrm{I})$ catalyst were slower and the enantioselectivities were lower. Solvent and temperature have measurable influence over enantioselectivity. It appears that dichloromethane and benzene are better solvents for high enantioselectivity. Ee values could be slightly improved at low temperature. On the other hand, the effect of the structure of thiols on the enantioselectivity was also studied (Table 1, entries 10,11,12). Although both aryl and aliphatic thiols were tested, the enantioselectivity was not improved. The optimization experiments concluded that the reaction with thiophenol in $\mathrm{CH}_{2} \mathrm{Cl}_{2}$ at $0{ }^{\circ} \mathrm{C}$ with catalyst 7 gives the best enantioselectivity (23\% ee, Table 1, entry 7). 


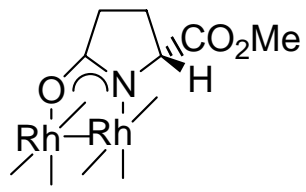

$4 \mathrm{Rh}_{2}(5 S-M E P Y)_{4}$

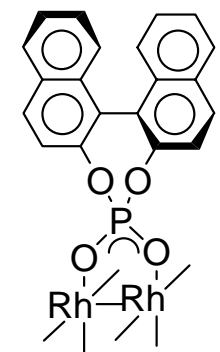

$5 \mathrm{Rh}_{2}(\mathrm{~S}-\mathrm{BNP})_{4}$

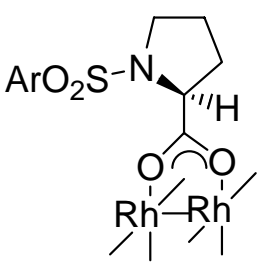

$6 \mathrm{Rh}_{2}(\mathrm{~S}-\mathrm{TBSP})_{4}\left(\mathrm{Ar}=4-{ }^{\mathrm{t}} \mathrm{BuC}_{6} \mathrm{H}_{4}\right)$

$7 \mathrm{Rh}_{2}(\mathrm{~S}-\mathrm{DOSP})_{4}\left(\mathrm{Ar}=4-\mathrm{CH}_{3}\left(\mathrm{CH}_{2}\right)_{10-12} \mathrm{C}_{6} \mathrm{H}_{4}\right)$<smiles>CC(C)(C)NC(=O)c1cccc(Cl)c1C=NC1CCCC[C@H]1N=Cc1c(Cl)cccc1Cl</smiles>

8<smiles>CC(C)(C)C1=N[C@@H](C(C)(C)C)CO1</smiles>

$\mathrm{Cu}(\mathrm{MeCN})_{4} \mathrm{PF}_{6}$

9

\section{Scheme 3}

Table 1. Enantioselectivity of the reaction of phenyldiazoacetate and thiols with chiral $\mathrm{Cu}(\mathrm{I})$ or $\mathrm{Rh}(\mathrm{II})$ catalysts

\begin{tabular}{llclcccc}
\hline Entry & Thiols 2 $(\mathrm{R}=)$ & Cat $^{\mathrm{a}}$ & Solvent & $\begin{array}{c}\text { Temp } \\
\left({ }^{\circ} \mathrm{C}\right)\end{array}$ & $\begin{array}{c}\text { Time } \\
(\mathrm{h})\end{array}$ & ee(\%) & $\begin{array}{c}\text { Yield } \\
(\%)^{\mathrm{c}}\end{array}$ \\
\hline 1 & $\mathrm{Ph}$ & $\mathbf{4}$ & $\mathrm{CH}_{2} \mathrm{Cl}_{2}$ & $\mathrm{rt}$ & 8 & 1 & 56 \\
2 & $\mathrm{Ph}$ & $\mathbf{5}$ & $\mathrm{CH}_{2} \mathrm{Cl}_{2}$ & $\mathrm{rt}$ & 4 & 13 & 49 \\
3 & $\mathrm{Ph}$ & $\mathbf{5}$ & $\mathrm{PhH}^{\mathrm{b}}$ & $\mathrm{rt}$ & 5 & 10 & 60 \\
4 & $\mathrm{Ph}$ & $\mathbf{5}$ & $\mathrm{CH}_{2} \mathrm{Cl}_{2}$ & 0 & 8 & 14 & 52 \\
5 & $\mathrm{Ph}$ & $\mathbf{5}$ & n-hexane & $\mathrm{rt}$ & 12 & 5 & 49 \\
6 & $\mathrm{Ph}$ & $\mathbf{6}$ & $\mathrm{CH}_{2} \mathrm{Cl}_{2}$ & 0 & 2 & 21 & 53 \\
7 & $\mathrm{Ph}$ & $\mathbf{7}$ & $\mathrm{CH}_{2} \mathrm{Cl}_{2}$ & 0 & 2 & 23 & 61 \\
8 & $\mathrm{Ph}$ & $\mathbf{8}$ & $\mathrm{CH}_{2} \mathrm{Cl}_{2}$ & $\mathrm{rt}$ & 12 & 7 & 45 \\
9 & $\mathrm{Ph}$ & $\mathbf{9}$ & $\mathrm{CH}_{2} \mathrm{Cl}_{2}$ & $\mathrm{rt}$ & 12 & 6 & 39 \\
10 & $2-\mathrm{ClPh}$ & $\mathbf{5}$ & $\mathrm{CH}_{2} \mathrm{Cl}_{2}$ & $\mathrm{rt}$ & 4 & 6 & 47 \\
11 & $2,6-(\mathrm{CH})_{2} \mathrm{Ph}$ & $\mathbf{5}$ & $\mathrm{CH}_{2} \mathrm{Cl}_{2}$ & $\mathrm{rt}$ & 4 & 5 & 62 \\
12 & Cyclohexanyl & $\mathbf{5}$ & $\mathrm{CH}_{2} \mathrm{Cl}_{2}$ & $\mathrm{rt}$ & 4 & 6 & 72 \\
\hline
\end{tabular}

${ }^{\mathrm{a}}$ For $\mathrm{Cu}(\mathrm{I})$ catalyst: chiral ligand (11 mol \%) was mixed with $\mathrm{Cu}(\mathrm{MeCN})_{4} \mathrm{PF}_{6}(10 \mathrm{~mol} \%)$; for $\mathrm{Rh}$ (II) catalyst: $0.5 \%$ mol catalyst is used. ${ }^{\mathrm{b}} \mathrm{Ee}$ 's determined by chiral HPLC; Chiracel OJ; hexane/iso-propanol. ' Isolated yields.

Although the enantioselectivity is rather low, we proceeded to apply the best reaction conditions in Table 1 to other aryldiazoacetates. Two Rh(II) catalysts, $\mathbf{5}$ and 7, were used, and the results are summarized in Table 2 . It demonstrates that moderately low enantioselectivity can be 
achieved in general with a series of aryl diazoacetates. We could observe a dependence of the enantioselectivity on the substituents in the phenyl ring of the aryldiazoacetate substrates. The Rh(II) catalyst 7 usually works better for the diazo compounds with para substituents in the phenyl ring, while the catalyst 5 appeared to be the opposite.

Table 2. Enantioselectivity of the reaction of aryldiazoacetate 1 and thiols $\mathbf{2}(\mathrm{R}=\mathrm{Ph})$ with chiral Rh(II) catalysts 5 and 7

\begin{tabular}{llcccc}
\hline Entry & $\begin{array}{c}\text { Diazo Compound } \mathbf{1} \\
(\mathrm{Ar})\end{array}$ & Cat $^{\mathrm{a}}$ & $\begin{array}{c}\text { Time } \\
(\mathrm{h})\end{array}$ & $\begin{array}{c}\text { Ee (\%) } \\
\text { b }\end{array}$ & $\begin{array}{c}\text { Yield }^{\mathrm{c}} \\
(\%)\end{array}$ \\
\hline 1 & $\mathrm{C}_{6} \mathrm{H}_{5}$ & $\mathbf{7}$ & 2 & 23 & 61 \\
2 & $p-\mathrm{MeOC}_{6} \mathrm{H}_{4}$ & $\mathbf{5}$ & 3 & 14 & 54 \\
3 & $p-\mathrm{MeOC}_{6} \mathrm{H}_{4}$ & 7 & 2 & 13 & 48 \\
4 & $m-\mathrm{ClC}_{6} \mathrm{H}_{4}$ & $\mathbf{5}$ & 4 & 14 & 62 \\
5 & $p-\mathrm{ClC}_{6} \mathrm{H}_{4}$ & 7 & 2 & 14 & 61 \\
6 & naphthyl & $\mathbf{5}$ & 4 & 12 & 63 \\
7 & $p-\mathrm{BrC}_{6} \mathrm{H}_{4}$ & $\mathbf{7}$ & 2 & 18 & 67 \\
\hline
\end{tabular}

${ }^{\mathrm{a}}$ For Rh(II) catalyst: $0.5 \% \mathrm{~mol}$ catalyst is used. ${ }^{\mathrm{b}}$ For $\mathrm{Rh}(\mathrm{II})$ catalyzed reaction, the temperature is $0{ }^{\circ} \mathrm{C}$. Ee's determined by chiral HPLC using the condition given in Table l. 'Isolated yields.

In contrast to transition metal-catalyzed carbene insertions into $\mathrm{C}-\mathrm{H}$ or $\mathrm{Si}-\mathrm{H}$ bonds, in which the catalytic asymmetric induction has reached high level, ${ }^{7,8}$ the corresponding insertions into polar $\mathrm{X}-\mathrm{H}$ bonds ( $\mathrm{X}=\mathrm{O}, \mathrm{S}, \mathrm{N}$, etc.) have proved to be much more difficult. ${ }^{9}$ This fact may reflect the difference in the reaction mechanism. The insertions into $\mathrm{C}-\mathrm{H}$ or $\mathrm{Si}-\mathrm{H}$ bonds follow concerted pathway, while the insertions into polar bonds are believed to follow stepwise process, in which ylide is firstly generated, followed by proton transfer (Scheme 4). ${ }^{10}$

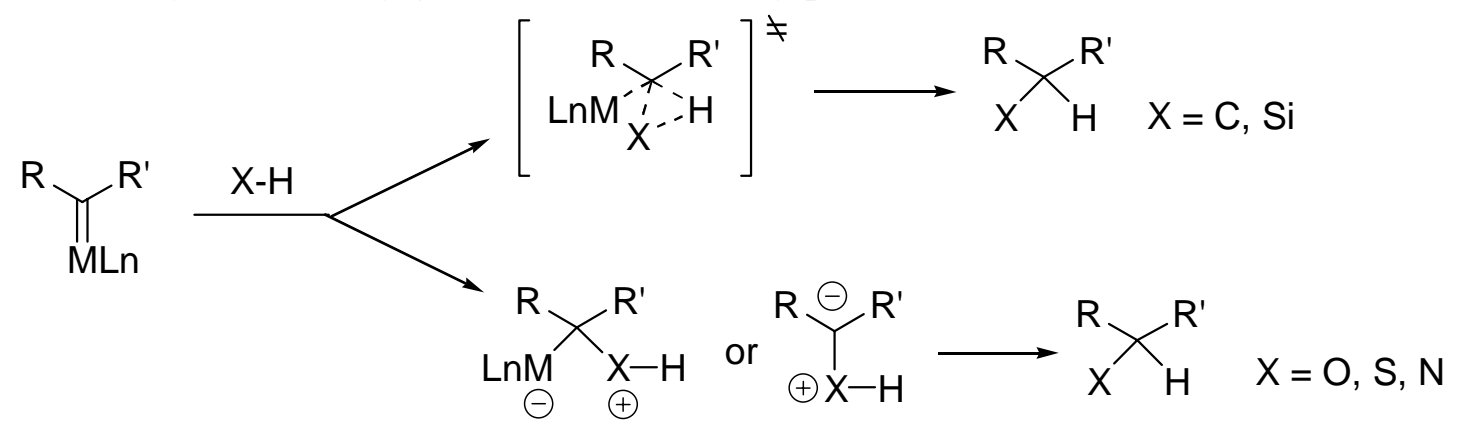

\section{Scheme 4}

In summary, we have conducted a systematic investigation on catalytic asymmetric S-H insertion generated from aryldiazoacetates. Unfortunately, the enantioselectivity is not dramatically improved compared with Brunner and Doyle's investigation. Further effort is needed to improve the enantioselectivity to the level that asymmetric S-H insertion reaction can be practically useful. 


\section{Experimental Section}

General Procedures. All reactions were performed under a nitrogen atmosphere in a flame-dried reaction flask, and the components were added via Syringe. All solvents were distilled prior to use. For chromatography, 100-200 mesh silica gel (Qindao, China) was employed. ${ }^{1} \mathrm{H}$ and ${ }^{13} \mathrm{C}$ NMR spectra were recorded at $300 \mathrm{MHz}$ and $75 \mathrm{MHz}$ with Varian Mercury 300 spectrometer. Chemical shifts are reported in ppm using tetramethylsilane as internal standard. IR spectra were recorded with a Nicolet 5MX-S infrared spectrometer. Mass spectra were obtained on a VG ZAB-HS mass spectrometer. Aryl diazoacetates ${ }^{11}$ and $\mathrm{Cu}(\mathrm{MeCN})_{4} \mathrm{PF}_{6}{ }^{12}$ was prepared according to literature procedure. Chiral bisoxazoline ligands, and chiral $\mathrm{Rh}(\mathrm{II})$ catalysts $\mathrm{Rh}_{2}(S \text {-TBSP })_{4} 6$ and $\mathrm{Rh}_{2}(S \text {-DOSP })_{4} 7$ were purchased from Aldrich. HPLC analysis was performed at HP 1100 apparatus with Chiracel OJ column.

\section{Typical procedure for the reaction of aryldiazoacetate with sulfide catalyzed by $\mathrm{Cu}(\mathrm{I})$ complex}

In nitrogen atmosphere, $\mathrm{Cu}(\mathrm{MeCN})_{4} \mathrm{PF}_{6}\left(6.25 \times 10^{-3} \mathrm{mmol}, 2.2 \mathrm{mg}\right)$ and ligand $9\left(9.4 \times 10^{-3}\right.$ $\mathrm{mmol}, 2.3 \mathrm{mg}$ ) were added to a $25 \mathrm{~mL}$ round-bottom flask. Dry dichloromethane $(2 \mathrm{~mL})$ was introduced and the solution was stirred for $1 \mathrm{~h}$. To the slightly blue solution was then added thiols $2\left(\mathrm{R}=\mathrm{C}_{6} \mathrm{H}_{4}, 1.25 \times 10^{-1} \mathrm{mmol}, 14 \mathrm{mg}\right)$ in dichloromethane $(1 \mathrm{~mL})$. The solution turned to light purple and remained homogenous. Methyl phenyldiazoacetate $\left(\mathbf{1}, \mathrm{Ar}=\mathrm{C}_{6} \mathrm{H}_{4}\right)\left(6.25 \times 10^{-2}\right.$ mmol, $11 \mathrm{mg})$ in dry dichloromethane $(10 \mathrm{~mL})$ was added via a syringe over $30 \mathrm{~min}$. The solution was stirred for additional $12 \mathrm{~h}$. Solvent was removed by evaporation and the green oily residue was purified by column chromatography (petroleum ether/ethyl acetate $=20: 1$ ) to give $3\left(\mathrm{Ar}=\mathrm{C}_{6} \mathrm{H}_{4}, \mathrm{R}=\mathrm{C}_{6} \mathrm{H}_{4}\right)$ as oil (6.3 mg, $\left.39 \%\right)$.

\section{Typical procedure for the reaction of aryldiazoacetate with sulfide catalyzed by $R h($ II) complex}

In nitrogen atmosphere, catalyst $7\left(3.13 \times 10^{-4} \mathrm{mmol}, 0.6 \mathrm{mg}\right)$ was added to a $25 \mathrm{~mL}$ round-bottom flask. Dry dichloromethane $(4 \mathrm{~mL})$ was introduced and the solution was stirred for $1 \mathrm{~h}$. To the slightly blue solution was then added thiol $2\left(\mathrm{Ar}^{\prime}=\mathrm{C}_{6} \mathrm{H}_{4}, 1.25 \times 10^{-1} \mathrm{mmol}, 14 \mathrm{mg}\right)$ in dichloromethane $(1 \mathrm{~mL})$. The solution turned to light purple and remained homogenous. The flask was put into an ice bath, then methyl phenyldiazoacetate $\left(\mathbf{1}, \mathrm{Ar}=\mathrm{C}_{6} \mathrm{H}_{4}\right)\left(6.25 \times 10^{-2} \mathrm{mmol}\right.$, $11 \mathrm{mg})$ in dry dichloromethane $(10 \mathrm{~mL})$ was added via a syringe over $30 \mathrm{~min}$. The solution was stirred for additional $2 \mathrm{~h}$. Solvent was removed by evaporation and the green oily residue was purified by column chromatography (petroleum ether/ethyl acetate $=20: 1)$ to give $3\left(\mathrm{Ar}=\mathrm{C}_{6} \mathrm{H}_{4}\right.$, $\left.\mathrm{R}=\mathrm{C}_{6} \mathrm{H}_{4}\right)$ as oil (9.8 mg, $\left.61 \%\right)$.

Methyl 2-thiophenyl-phenylacetate (3, $\left.\mathbf{A r}=\mathbf{C}_{6} \mathbf{H}_{5}, \mathbf{R}=\mathbf{C}_{6} \mathbf{H}_{5}\right)$. IR $\left(\mathrm{CDCl}_{3}\right) 1738$ (s); ${ }^{1} \mathrm{H}$ NMR $\left(300 \mathrm{MHz}, \mathrm{CDCl}_{3}\right) \delta 3.61$ (s, 3H), $4.91(\mathrm{~s}, 1 \mathrm{H}), 7.20-7.44(\mathrm{~m}, 10 \mathrm{H}) ;{ }^{13} \mathrm{C} \mathrm{NMR}\left(75 \mathrm{MHz}, \mathrm{CDCl}_{3}\right)$ $\delta$ 52.53, 56.10, 127.85, 128.17, 128.33, 128.52, 128.71, 128.82, 128.94, 129.01, 129.07, 132.43, 133.49, 135.40, 170.69; MS ( $\mathrm{m} / \mathrm{z}$, relative intensity) $258\left(\mathrm{M}^{+}, 49\right), 199$ (44), 184 (8), 150 (18), 149 (27), 121 (30), 86 (100), 84 (100), 47 (100), 35 (98); HRMS calcd for $\mathrm{C}_{15} \mathrm{H}_{14} \mathrm{O}_{2} \mathrm{~S}: 258.0714$, found 258.0708. HPLC (254 nm) $t_{R}=20.957 \mathrm{~min}, t_{R}=40.150 \mathrm{~min}$. 
Methyl 2-(2-chloro)thiophenyl-phenylacetate (3, Ar $\left.=\mathbf{C}_{6} \mathbf{H}_{5}, \mathbf{R}=\mathbf{o}-\mathbf{C l C}_{\mathbf{6}} \mathbf{H}_{4}\right)$. IR $\left(\mathrm{CDCl}_{3}\right) 1738$ (s); ${ }^{1} \mathrm{H}$ NMR (300 MHz, $\left.\mathrm{CDCl}_{3}\right) \delta 3.66$ (s, 3H), 5.07 (s, 1H), 7.11-7.48 (m, 9H); ${ }^{13} \mathrm{C}$ NMR (75 $\left.\mathrm{MHz} \mathrm{CDCl}_{3}\right) \delta 52.75,54.18,127.12,127.25,128.41,128.68,128.84,128.99,129.14,129.82$, 133.13, 134.82, 170.41; MS (m/z, relative intensity) 292 ( $\left.\mathrm{M}^{+}, 17\right), 233$ (32), 197 (8), 150 (85), 149 (44), 121 (52), 91 (100), 65 (42), 39 (27); HRMS calcd for $\mathrm{C}_{15} \mathrm{H}_{13} \mathrm{O}_{2} \mathrm{SCl}$ : 292.0324, found 292.0317. HPLC (254 nm) $t_{R}=32.876 \mathrm{~min}, t_{R}=56.907 \mathrm{~min}$.

Methyl 2-thiophenyl-p-methoxylphenylacetate (3, Ar $=p$ - $\left.\mathbf{M e O C}_{6} \mathbf{H}_{4}, \mathbf{R}=\mathbf{C}_{6} \mathbf{H}_{5}\right) . \mathrm{IR}\left(\mathrm{CDCl}_{3}\right)$ 1737 (s); ${ }^{1} \mathrm{H}$ NMR (300 MHz, $\left.\mathrm{CDCl}_{3}\right) \delta 3.66$ (s, 3H), 3.79 (s, 3H), 4.88 (s, 1H), 6.83-6.86 (m, 2H), 7.24-7.27 (m, 4H), 7.35-7.38 (m, 3H); $\left.{ }^{13} \mathrm{C} \mathrm{NMR} \mathrm{(75} \mathrm{MHz,} \mathrm{CDCl}_{3}\right) \delta$ 52.51, 55.09, 55.47, 113.92, 114.11, 127.30, 127.79, 128.83, 129.55, 132.39, 133.68, 170.04; MS (m/z, relative intensity) 288 ( $\left.\mathrm{M}^{+}, 8\right), 229$ (37), 228 (6), 179 (100), 151 (88), 121 (40), 110 (27), 77 (23), 51 (18); HRMS calcd for $\mathrm{C}_{16} \mathrm{H}_{16} \mathrm{O}_{2} \mathrm{~S}: 288.0820$, found 288.0813. HPLC (254 nm) $t_{R}=33.555 \mathrm{~min}, t_{R}=$ $45.345 \mathrm{~min}$.

Methyl 2-thiophenyl-p-chlorophenylacetate (3, Ar = p- $\left.\mathbf{C l C}_{\mathbf{6}} \mathbf{H}_{\mathbf{4}}, \mathbf{R}=\mathbf{C}_{\mathbf{6}} \mathbf{H}_{5}\right)$. IR $\left(\mathrm{CDCl}_{3}\right) 1738$ (s); ${ }^{1} \mathrm{H}$ NMR (300 MHz, $\mathrm{CDCl}_{3}$ ) $\delta 3.67$ (s, 3H), 4.85 (s, 1H), 7.25-7.37 (m, 9H); ${ }^{13} \mathrm{C}$ NMR (75 $\left.\mathrm{MHz} \mathrm{CDCl}_{3}\right) \delta$ 52.74, 55.56, 128.24, 128.73, 128.97, 129.81, 132.93, 134.13, 170.41; MS (m/z, relative intensity) $292\left(\mathrm{M}^{+}, 50\right), 233$ (67), 183 (99), 155 (100), 139 (14), 109 (22), 65 (18), 39 (14); HRMS calcd for $\mathrm{C}_{15} \mathrm{H}_{13} \mathrm{O}_{2} \mathrm{SCl}$ : 292.0324, found 292.0323. HPLC (254 nm) $t_{R}=13.507 \mathrm{~min}$, $t_{R}=17.173 \mathrm{~min}$.

Methyl 2-(2,6-dimethyl)thiophenyl-phenylacetate (3, Ar $\left.=\mathrm{C}_{6} \mathrm{H}_{5}, \mathbf{R}=\mathbf{2 , 6}-\left(\mathrm{CH}_{3}\right)_{2} \mathrm{C}_{6} \mathrm{H}_{3}\right)$. IR $\left(\mathrm{CDCl}_{3}\right) 1732$ (s); ${ }^{1} \mathrm{H}$ NMR $\left(300 \mathrm{MHz}, \mathrm{CDCl}_{3}\right) \delta 2.35$ (s, 6H), 3.51 (s, 3H), $4.50(\mathrm{~s}, 1 \mathrm{H})$, 6.98-7.04 (m, 3H), 7.21-7.22 (m, 3H), 7.30-7.33 (m, 2H); ${ }^{13} \mathrm{C}$ NMR (75 MHz, $\left.\mathrm{CDCl}_{3}\right) \delta 21.76$, 29.661, 52.41, 55.02, 128.14, 128.40, 128.46, 129.06, 131.43, 136.04, 143.81, 170.99; MS (m/z, relative intensity) 286 ( $\mathrm{M}^{+}$, 51), 254 (11), 227 (41), 150 (10), 149 (94), 121 (100), 91 (69), 57 (53), 43 (39); HRMS calcd for $\mathrm{C}_{17} \mathrm{H}_{18} \mathrm{O}_{2} \mathrm{~S}$ : 286.1027, found 286.1030. HPLC (254 nm) $t_{R}=$ $16.681 \mathrm{~min}, t_{R}=32.046 \mathrm{~min}$.

Methyl 2-cyclohexanyl -phenylacetate (3, Ar $\left.=\mathbf{C}_{6} \mathbf{H}_{5}, \mathbf{R}=\mathbf{C}_{6} \mathbf{H}_{11}\right)$. IR $\left(\mathrm{CDCl}_{3}\right) 1738$ (s); ${ }^{1} \mathrm{H}$ NMR (300 MHz, $\left.\mathrm{CDCl}_{3}\right) \delta$ 1.22-1.36 (m, 5H), 1.56-1.72 (m, 1H), 1.91-1.95 (m, 2H), 1.95-1.97 (m, 2H), 2.30-2.66 (m, 1H), 3.72 (s, 3H), $4.66(\mathrm{~s}, 1 \mathrm{H}), 7.23-7.48(\mathrm{~m}, 5 \mathrm{H}) ;{ }^{13} \mathrm{C} \mathrm{NMR}(75 \mathrm{MHz}$, $\left.\mathrm{CDCl}_{3}\right) \delta$ 25.609, 25.738, 33.126, 43.970, 50.678, 52.632, 127.964, 128.330, 128.552, 136.504, 171.686; MS (m/z, relative intensity) $265\left[(\mathrm{M}+1)^{+}, 50\right], 184$ (6), 150 (72), 149 (18), 123 (100), 91 (20), 77 (18), 55 (37), 41 (28); HRMS calcd for $\mathrm{C}_{15} \mathrm{H}_{20} \mathrm{O}_{2} \mathrm{~S}: 264.1184$, found 264.1193. HPLC $(254 \mathrm{~nm}) t_{R}=9.973 \mathrm{~min}, t_{R}=18.603 \mathrm{~min}$.

Methyl 2-thiophenyl-1-naphthylacetate (3, Ar = Naphthyl, $\left.\mathbf{R}=\mathbf{C}_{\mathbf{6}} \mathbf{H}_{\mathbf{5}}\right)$. IR $\left(\mathrm{CDCl}_{3}\right) 1739$ (s); ${ }^{1} \mathrm{H}$ NMR (300 MHz, $\mathrm{CDCl}_{3}$ ) $\delta 3.66$ (s, 3H), 5.67 (s, 1H), 7.22-7.87 (m, 11H), 8.13-8.16 (m, 1H); ${ }^{13} \mathrm{C} \mathrm{NMR}\left(75 \mathrm{MHz}, \mathrm{CDCl}_{3}\right) \delta 52.99,57.14,123.04,125.32,125.85,126.14,126.66,126.79$, 127.91, 128.25, 128.97, 130.84, 131.14, 132.36, 133.89, 134.10, 171.22; MS (m/z, relative intensity) 308 ( $\mathrm{M}^{+}$, 39), 249 (21), 200 (36), 199 (100), 171 (77), 141 (40), 110 (14), 63 (9), 47 (57); HRMS calcd for $\mathrm{C}_{19} \mathrm{H}_{16} \mathrm{O}_{2} \mathrm{~S}$ : 308.0871, found 308.0861. HPLC (254 nm) $t_{R}=45.579 \mathrm{~min}$, $t_{R}=55.289 \mathrm{~min}$.

Methyl 2-thiophenyl-m-chlorophenylacetate (3, $\left.\mathbf{A r}=\mathbf{m}-\mathbf{C l C}_{\mathbf{6}} \mathbf{H}_{4}, \mathbf{R}=\mathbf{C}_{\mathbf{6}} \mathbf{H}_{5}\right)$. IR $\left(\mathrm{CDCl}_{3}\right) 1738$ (s); ${ }^{1} \mathrm{H}$ NMR (300 MHz, $\left.\mathrm{CDCl}_{3}\right) \delta 3.68$ (s, 3H), 4.84 (s, 1H), 7.23-7.43 (m, 9H); ${ }^{13} \mathrm{C}$ NMR (75 ISSN 1551-7012 
$\left.\mathrm{MHz}, \mathrm{CDCl}_{3}\right) \delta 52.83,55.80,126.69,128.34,128.46,128.59,129.03,129.80,132.94,132.99$, 134.42, 137.54, 170.28; MS (m/z, relative intensity) $292\left(\mathrm{M}^{+}, 33\right), 233$ (100), 183 (21), 155 (52), 125 (10), 110 (17), 91 (30), 65 (22), 39 (10); HRMS calcd for $\mathrm{C}_{15} \mathrm{H}_{13} \mathrm{O}_{2} \mathrm{SCl}$ : 292.0324, found 292.0317. HPLC (254 nm) $t_{R}=15.237 \mathrm{~min}, t_{R}=19.157 \mathrm{~min}$.

Methyl 2-thiophenyl-p-Bromephenylacetate (3, $\left.\mathrm{Ar}=\boldsymbol{p}-\mathrm{BrC}_{\mathbf{6}} \mathbf{H}_{4}, \mathbf{R}=\mathbf{C}_{\mathbf{6}} \mathbf{H}_{5}\right)$. IR $\left(\mathrm{CDCl}_{3}\right) 1738$ (s); ${ }^{1} \mathrm{H}$ NMR (300 MHz, $\left.\mathrm{CDCl}_{3}\right) \delta 3.66$ (s, 3H), 4.84 (s, 1H), 7.26-7.44 (m, 9H); ${ }^{13} \mathrm{C}$ NMR (75 $\left.\mathrm{MHz} \mathrm{CDCl}_{3}\right) \delta 52.74,55.66,122.36,128.26,129.00,129.54,130.13,131.71,132.94,134.70$, 170.32; MS (m/z, relative intensity) $336\left(\mathrm{M}^{+}, 2\right), 279$ (2), 264 (14), 229 (5), 227 (5), 205 (65), 199 (5), 183 (5), 155 (8), 139 (5), 124 (6), 100 (3), 89 (44), 85 (13), 71 (34), 43 (100), 41 (76), 27 (35); HRMS calcd for $\mathrm{C}_{15} \mathrm{H}_{13} \mathrm{O}_{2} \mathrm{SBr}$ : 335.9819, found 335.9824. HPLC (254 nm) $t_{R}=15.567$ $\min , t_{R}=21.933 \mathrm{~min}$.

\section{Acknowledgments}

The project is generously supported by Natural Science Foundation of China (Grant No. 20172002, 20225205), State Key Laboratory of Elemento-Organic Chemistry of Nankai University and by Trans-Century Training Programme Foundation for the Talents by Ministry of Education of China.

\section{References}

1. (a) Bohacek, R.; De Lombaert, S.; McMartin, C.; Priestle, J.; Grutter, M. J. Am. Chem. Soc. 1996, 118, 8231; (b) Robl, J. A.; Sun, C. -Q.; Stevenson, J.; Ryono, D. E.; Simpkins, L. M.; Cimarusti, M. P.; Dejneka, T.; Slusarchyk, W. A.; Chao, S.; Stratton, L.; Misra, R. N.; Bednarz, M. S.; Asaad, M. M.; Cheung, H. S.; Abboa-Offei, B. E.; Smith, P. L.; Mathers, P. D.; Fox, M.; Schaeffer, T. R.; Seymour, A. A.; Trippodo, N. C. J. Med. Chem. 1997, 40, 1570; (c) Gaucher, J. F.; Selkti, M.; Tiraboschi, G.; Prange, T.; Roques, B. P.; Tomas, A.; Fournie-Zaluski, M. C. Biochemistry 1999, 38, 12569.

2. For recent examples, see: (a) Nam, J.; Lee, S.-k.; Kim, K. Y.; Park, Y. S. Tetrahedron Lett. 2002, 43, 8253. (b) Schedel, H.; Quaedflieg, P. J. L. M.; Broxterman, Q. B.; Bisson, W.; Duchateau, A. L. L.; Maes, I. C. H.; Herzschuh, R.; Burger, K. Tetrahedron: Asymmetry 2000, 11, 2125. (c) Chen, B. -C.; Bednarz, M. S.; Kocy, O. R.; Sundeen, J. E. Tetrahedron: Asymmetry 1998, 9, 1641.

3. (a) McKervey, M. A.; Ratananukul, P. Tetrahedron Lett. 1982, 23, 2509. (b) Moyer, M. P.; Feldman, P. L.; Rapoport, H. J. Org. Chem. 1985, 50, 5223. (c) Moody, C. J.; Taylor, R. J. Tetrahedron Lett. 1987, 28, 5351.

4. Brunner, H.; Wutz, K.; Doyle, M. P. Monatsh. Chem. 1990, 121, 755.

5. For a review, see: Davies, H. M. L.; Antoulinakis, E. G. J. Organomet. Chem. 2001, 617-618, 47.

6. Zhang, X.; Qu, Z.; Ma, Z.; Shi, W.; Jin, X.; Wang, J. J. Org. Chem. 2002, 67, 5621.

7. Doyle, M. P.; Forbes, D. C. Chem. Rev. 1998, 98, 911.

8. Sulikowski, G. A.; Cha, K. L.; Sulikowski, M. M. Tetrahedron: Asymmetry 1998, 3145. 
9. (a) Ferris, L.; Haigh, D.; Moody, C. J. Tetrahedron Lett. 1996, 37, 107. (b) Buck, R. T.; Moody, C. J.; Pepper, A. G. Arkivoc 2002, 16; (c) Garcia, C. F.; McKervey, M. A.; Ye, T. J. Chem. Soc., Chem. Commun. 1996, 1465.

10. Miller, D. J.; Moody, C. J. Tetrahedron 1995, 51, 10811.

11. Qu, Z.; Shi, W.; Wang, J. J. Org. Chem. 2001, 66, 8139 and references cited therein.

12. Kubas, G. J. Inorg. Synth. 1979, 19, 90. 